\title{
Inverse Problem in Space Fractional Advection Diffusion Equation
}

\author{
Gisele Moraes Marinho ${ }^{1}$ \\ Universidade do Estado do Rio de Janeiro, Instituto Politécnico \\ Jader Lugon Júnior ${ }^{2}$ \\ Instituto Federal Fluminense \\ Diego Campos Knupp ${ }^{3}$ \\ Antônio J. Silva Neto ${ }^{4}$ \\ Joao Flávio Vieira Vasconcellos ${ }^{5}$ \\ Universidade do Estado do Rio de Janeiro, Instituto Politécnico
}

\begin{abstract}
This work studies an inverse problem of determining the fractional order, a diffusion coefficient and the average velocity simultaneously in one-dimensional space fractional advection-diffusion equation. The forward problem is performed by an implicit finite difference scheme. Here, the solution of the inverse problem is performed in R environment using the Generalized Simulated Annealing algorithm (GenSA) package. Numerical experiments show that the algorithm is able to retrieve the parameters with good accuracy.
\end{abstract}

Key-words. Fractional Diffusion, Inverse Problems, Simultaneous Inversion, GenSA

\section{Introduction}

For many decades diffusion processes were described by Fick's law and its generalizations, however this approach cannot describe transport phenomena in which particles spreads faster or slower than the predicted by the classical models. This class of processes are referred to as anomalous diffusion, and in the last two decades has been generating increasing interest in many fields, as biology [11], medicine [16] and finance [1].

In this context, fractional diffusion equations have shown be quite efficient for mathematical modeling such anomalous transport process by replacing traditional integer order derivatives with fractional derivative.

Inverse modeling of such kind of equation is important, as it can provide an estimate of parameters that in many cases cannot be measured using direct modeling. Due to the

\footnotetext{
${ }^{1}$ gmarinho@iprj.uerj.br

${ }^{2}$ jljunior@iff.edu.br

${ }^{3}$ diegoknupp@iprj.uerj.br

4 ajsneto@iprj.uerj.br

5 jflavio@iprj.uerj.br
} 
the fast growth of fractional calculus and the large number of phenomena represented by the fractional diffusion equations, inverse problems involving anomalous diffusion models have been investigated in several recent publications, as can be verified in [4], [7] and [13].

In general, inverse problems involving differential equations of fractional order seek to estimate the parameters of the model, such as diffusion coefficient and advection, the fractional order of the equations or even source terms.

This paper consider the analysis of simultaneous inversion of the fractional order, the diffusion coefficients and the average velocity in the following space fractional advectiondiffusion equation, SFADE:

$$
\frac{\partial u}{\partial t}=D \frac{\partial^{\alpha} u}{\partial x^{\alpha}}-v \frac{\partial u}{\partial x}+g(x, t), \quad 0 \leq x \leq L, \quad 0 \leq t \leq t_{f}
$$

subject to the initial and boundary conditions given by:

$$
\begin{gathered}
u(x, 0)=f(x) \\
u(0, t)=0, \quad u(L, t)=0
\end{gathered}
$$

where $u(x, t)$ represents, for example, a solute concentration, $1<\alpha<2$ is the order of the fractional derivative, $D$ is the diffusion coefficient, $v>0$ is the average velocity, while $g(x, t)$ is a external source and $\frac{\partial^{\alpha} u}{\partial x^{\alpha}}$ is the Riemann-Liouville fractional derivative in space of $u$.

At first, a sensitivity analysis of parameters is performed in order to verify the possibility of estimating the three parameters of the model simultaneously. Then, Generalized Simulated Anealing algorithm is used for the solution of the inverse problem.

\section{The Foward Problem}

The implicit finite difference schemes were employed for solving the foward problem. We consider a space domain $[0, L]$ that is discretised with $M+1$ uniformly spaced nodes $x_{i}=i \times h, i=0, \ldots, M$, with the spatial step $h=L / N$ and time time $\Delta t=t_{f} / N$. The space frational derivative are approximated using the Shifted Grünwald formula [8]:

$$
\frac{\partial^{\alpha} u}{\partial x^{\alpha}}=\frac{1}{h^{\alpha}} \sum_{k=0}^{i+1} \frac{\Gamma(k-\alpha)}{\Gamma(-\alpha) \Gamma(k+1)} u_{i-k+1}^{n+1}
$$

the first order derivative in Eq. (1a) is approximated using second order central differences and the the temporal derivative by the standard first order backward difference, or implicit Euler method. Defining $u_{i}^{n} \approx u\left(x_{i}, t_{n}\right)$ as the numerical solution, we obtain the implicit scheme:

$$
\frac{u_{i}^{n+1}-u_{i}^{n}}{\Delta t}=+D \frac{1}{h^{\alpha}} \sum_{k=0}^{i+1} \frac{\Gamma(k-\alpha)}{\Gamma(-\alpha) \Gamma(k+1)} u_{i-k+1}^{n+1}-v\left(\frac{u_{i}^{n+1}-u_{i-1}^{n+1}}{h}\right)+g_{i}^{n+1}
$$


Defining:

$$
q=\frac{D \Delta t}{h^{\alpha}}, \quad p=\frac{v \Delta t}{h}, \quad d_{k}=\frac{\Gamma(k-\alpha)}{\Gamma(-\alpha) \Gamma(k+1)}
$$

together with initial and boundary conditions, Eqs. (1b-d), the numerical schemes become:

$$
u_{i}^{n+1}-u_{i}^{n}=-p u_{i}^{n+1}+p u_{i-1}^{n+1}+q \sum_{k=0}^{i+1} d_{k} u_{i-k+1}^{n+1}+\Delta t g_{i}^{n+1}
$$

which can be written in the matrix form $A u^{n+1}=u^{n}+\Delta t g^{n+1}$, with $A=\left(a_{i j}\right)_{M-1 \times M-1}$ and coefficientes $a_{i j}$ for $i=1, \ldots M-2$ and $i=M-1$, given, respectively, by:

$$
a_{i j}=\left\{\begin{array}{ll}
0, & j>i+1 \\
-q d_{0}, & j=i+1, \\
1+p-q d_{1}, & j=i \\
-p-q d_{2}, & j=i-1 \\
-q d_{i-j+1}, & j<i-1
\end{array} \quad, \quad a_{M-1, j}= \begin{cases}-q d_{M-j}, & j=1, \ldots M-3 \\
-p-q d_{2}, & j=M-2 \\
1+p-q d_{1}, & j=M-1\end{cases}\right.
$$

The stability and convergence of the above difference scheme are proved in [3]. Here, the problem given in Eq. (5) is solved with a $\mathrm{C}$ routine.

\section{The Inverse Problem}

For investigating the inverse problem solution concerning the estimation of the three model parameters, $\mathbf{P}=\{\alpha, D, V\}$, we consider a vector of experimental data $\mathbf{Y}=$ $\left\{u_{1}^{e x p}, u_{1}^{e x p}, \ldots, u_{N d}^{e x p}\right\}$, simulated with the solution of Eq. (5) and with the addition of noise generated from a normal distribution with zero mean and known variance:

$$
Y_{i}=u_{i}\left(P_{\text {exact }}\right)+\epsilon_{i}, \quad \epsilon \approx N\left(0, \sigma_{i}^{2}\right)
$$

where $u_{i}^{e x p}$ are the experimental data in one or more positions and in different times, and $N d$ is the number of experimental data available.

\subsection{Generalized Simulated Annealing}

The Generalized Simulated Annealing introduced by [12] is a stochastic optimization algorithm based on the classical Simulated Annealing [5] and on the Fast Simulated Annealing [14]. This method has been applied in different problems such as epidemic models [6], protein folding [2] and optmization for inversion of first-arrival [9].

Basically, in GenSA candidate solutions are generated iteratively following a generalized Gaussian distribution and are accepted or not according to a Metropolis-like criterion. The stochasticity of both the generation of solutions and the acceptance criterion are controlled by the "temperature", which is gradually decreased through a predefined cooling schedule [6].

The generalized simulated annealing can then be summarized in three stages: 
1. The probability distribution function or the distribution of visitation: GSA uses a distorted Cauchy-Lorentz visiting distribution;

2. The probability of acceptance: a generalized Metropolis algorithm is used for the acceptance probability;

3. The programming of cooling or reduction of temperature.

The mathematical expressions for the three stages are detailed in [12].

In this work, the inverse problem are performed in $\mathrm{R}$ environment [10], using the package GenSA [15] that provides an implementation of Generalized Simulated Anealing algorithm. The GenSA R Package, it is used into a new $\mathrm{R}$ package, still in development, for solving inverse problems. The developed routines allow the evaluation of the sensitivity coefficients and the solution of the inverse problem using $\mathrm{R}$ packages for solving optimization problem. It is also able to call a external routine developed in other languages for the direct problem solution, such as the $\mathrm{C}$ routine used in this work.

One of benefits of using $\mathrm{R}$ language and environment is that it enables rapid prototyping of algorithms, access to a wide range of tools for statistical modeling, and the ability to easily generate customized plots of results.

\subsection{Sensitivity Analysis}

For the sensitivity analysis it was considered the scaled sensitivity coefficients that allows a more evident comparisons between the sensitivity coefficients with respect to different parameters and identification of linear dependence. The scaled sensitivity coefficients are obtained by multiplying the sensitivity coefficient by an estimate or reference value of the each parameter $\bar{X}_{P_{i}}=P_{i} \frac{\partial u}{\partial P_{i}}$ :

$$
\bar{X}_{\alpha}(x, t)=P_{\alpha} \frac{\partial u}{\partial \alpha}, \quad \bar{X}_{D}(x, t)=P_{D} \frac{\partial u}{\partial D}, \quad \bar{X}_{v}(x, t)=P_{v} \frac{\partial u}{\partial v}
$$

The first order derivatives are approximated by centered finite differences:

$$
\bar{X}_{P_{i}}=P_{i} \frac{u_{P_{i}+\delta}(x, t)-u_{P_{i}-\delta}(x, t)}{2 \delta}, \quad i=1, \ldots N d
$$

where $\delta$ represents a small pertubation in the parameter that is being analysed.

\section{Results and Discussions}

\subsection{Sensitivity Analysis}

It was considered a test case with $\alpha=1.8, D=1.0$ and $v=0.6$. Figure 1 illustrates the scaled sensitivity coefficients at $t=1$ and in the spatial domain $0 \leq x \leq \pi$.

After the sensitivity analysis it is possible to select the spatial positions where the transient values of the sensitivity coefficients were predominantly larger and therefore 
becoming the preferred location for the installation of a possible sensor for the collection of experimental data.

From the graphs analysis, it can be noticed that the sensitivity coefficients of parameters $v$ and $D$ are relatively small compared to the fractional order $\alpha$. However it was not possible to determine the best position for performing transient measurements concerning the inverse problem solution only by observing Figure 1 . Thus, it was investigated the sensitivity coefficients on time domain for different positions and the chosen was $x=3 \pi / 20$.

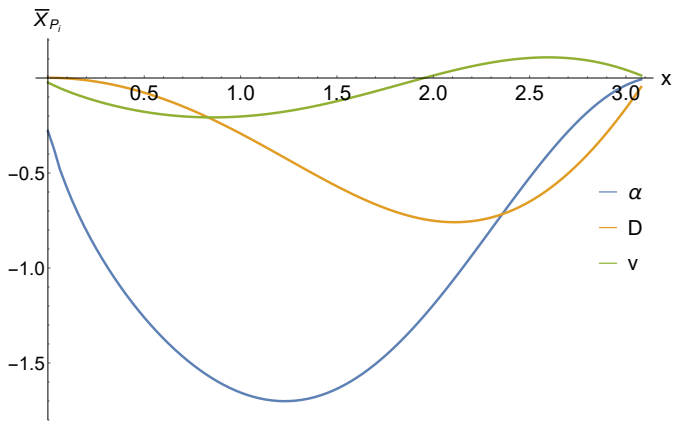

Figure 1: Sensitivity coefficients along the spatial domain at $t=1$.

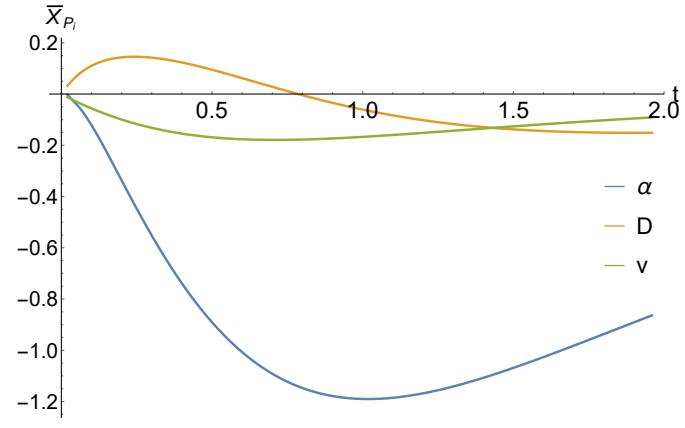

Figure 2: Sensitivity coefficients along the time domain at $x=3 \pi / 20$.

\subsection{Inverse Problem}

For the solution of the inverse problem it was considered the following values: $\alpha=1.8$, $D=1.0$ and $v=0.6$. For the inverse problem solution were employed 50 experimental data from $t=0$ to $t=1$ using a single sensor located at $x=3 \pi / 20$.

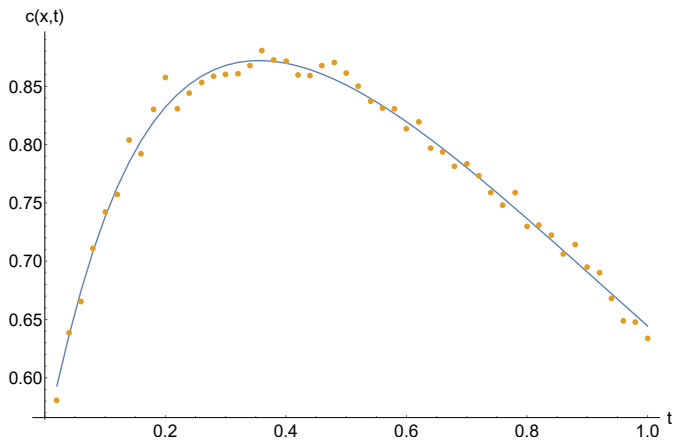

Figure 3: Simulated experimental data (dots) with $\sigma=0.0087$.

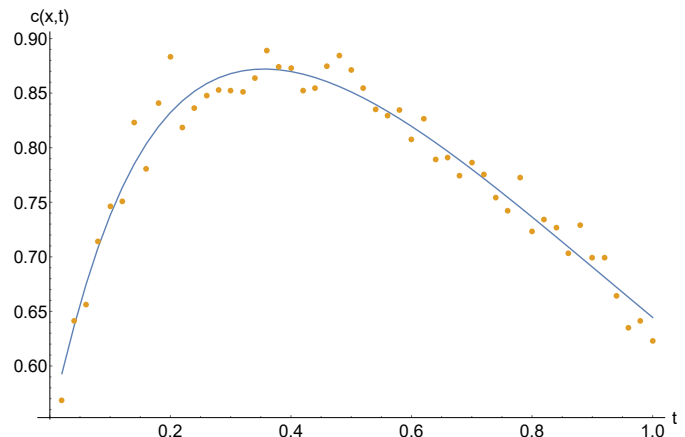

Figure 4: Simulated experimental data (dots) with $\sigma=0.021$.

Figures 3 and 4 show a set of experimental data at a single sensor $x=3 \pi / 20$, simulated by employing Eq. (7) with $\sigma=0.0087$ and $\sigma=0.021$, equivalent to $1 \%$ and $2.5 \%$ of the maximum value in the data. The blue curve shows the numerical solution employed to simulate the experimental data. 
The initial values for the parameters employed were $\mathbf{P}_{i n i}=\{1.7,0.4,0.2\}$, the lower and upper bounds for the parameters were chosen as $\mathbf{P}_{\text {lower }}=\{1.4,0.1,0.1\}$ and $\mathbf{P}_{\text {upper }}=$ $\{1.99,3.0,1.2\}$. The maximum running time of GenSA was fixed in 8 seconds and for the other control arguments it was used the internal default values.

The cost function is defined as the sum of the residuals:

$$
\text { cost }=\sum\left(Y_{i}-u_{i}^{\text {calc }}\right)^{2}, \quad i=1, \ldots 50
$$

where $Y_{i}$ is the vector with experimental data and $u_{i}^{\text {calc }}$ is the vector with calculated values at each iteration of the method.

The inverse problem solution 1 is presented in Table 1 . The results show that the errors present in the inputs amplify the estimates of the parameters, however the inversion solution is satisfactory even in the case of $2.5 \%$ noise level.

Table 1: Inverse problem results for

\begin{tabular}{cccccc}
\hline noise & $\alpha$ & $D$ & $v$ & Iter & cost \\
\hline- & 1.799996 & 1.000005 & 0.600001 & 351 & $1.06 \times 10^{-12}$ \\
$1 \%$ & 1.797359 & 1.007327 & 0.606635 & 400 & $3.36 \times 10^{-3}$ \\
$2.5 \%$ & 1.796836 & 1.012072 & 0.609231 & 386 & $1.4 \times 10^{-2}$ \\
\hline
\end{tabular}

\section{Conclusions}

The inverse problem formulation and solution for three parameters were investigated in relation to a space fractional advection-diffusion equation. The inverse analysis was carried out for transient measured experimental data obtained with a single sensor and, for the considered case in this work, it was possible to estimate simultaneously with good accuracy all three parameters that characterizes the model. In future works it is interesting to investigate the application of the approach proposed in this work for the solution of other types of inverse problems in anomalous diffusion equations, like the estimation of the orders of the fractional derivatives in space-time fractional diffusion from real data. Moreover, besides GenSA other $\mathrm{R}$ packages for solving optimization problems will be tested, in order to develop a practical tool for solving this class of problem.

\section{Acknowledgements}

This study was financed in part by the Coordenação de Aperfeiçoamento de Pessoal de Nível Superior - Brasil (CAPES) - Finance Code 001.

The authors also acknowledge the support provided by FAPERJ and CNPq.

\section{References}

[1] J. Blackledge. Application of the Fractional Diffusion Equation for Predicting Market Behaviour, International Journal of Applied Mathematics, 40(3):130-158, 2010. 
[2] U.H.E. Hansmann and Y. Okamoto. Comparative study of multicanonical and simulated annealing algorithms in the protein folding problem, Physica A: Statistical Mechanics and its Applications, 212(3-4):415-437, 1994.

[3] X. Jia, G. Li, C. Sun and D. Du. Simultaneous inversion for a diffusion coefficient and a spatially dependent source term in the SFADE, Inverse Problems in Science and Engineering, 24:832-859, 2016.

[4] B. Jin and W. Rundell. A tutorial on inverse problems for anomalous diffusion processes, Inverse Problems, 31(3), 2015.

[5] S. Kirkpatrick, C. D. Gelatt Jr. and M. P. Vecchi. Optimization by Simulated Annealing, Science, 220:671-680, 1983.

[6] O. H. Menin and C. Bauch. Solving the patient zero inverse problem by using generalized simulated annealing, Physica A: Statistical Mechanics and its Applications, 490:1513-1521, 2018.

[7] L. Miller and M. Yamamoto. Coefficient inverse problem for a fractional diffusion equation, Inverse Problems, 29(7):075013, 2013.

[8] I. Podlubny. Fractional differential equations: an introduction to fractional derivatives, fractional differential equations, to methods of their solution and some of their applications. Mathematics in science and engineering, San Diego, 1999.

[9] S. K. Pullammanappallil and J. N. Louie. A generalized simulated-annealing optimization for inversion of first-arrival times, Bulletin of the Seismological Society of America, 84(5):1397-1409, 1994.

[10] R Core Team. R: A Language and Environment for Statistical Computing, R Foundation for Statistical Computing, Vienna, Austria, 2014, http://www.R-project.org.

[11] C. Riedel et al. The heat released during catalytic turnover enhances the diffusion of an enzyme, Nature, 517:227-230, 2015.

[12] C. Sallis and D. Stariolo. Generalized Simulated Annealing, Physica A: Statistical Mechanics and its Applications, 233:395-406, 1996.

[13] L. G. Silva et al. Inverse problem in anomalous diffusion with uncertainty propagation, Computer Assisted Methods in Engineering and Science, 21:245-255, 2014.

[14] H. Szu and R. Hartley. Fast simulated annealing. Physics Letters A, 122:157-162, 1987.

[15] Y. Xiang, S.Gubian, B. Suomela and J. Hoeng. Generalized Simulated Annealing for Global Optimization: The GenSA Package, The R Journal, 5:13-28, 2013.

[16] F. Xiao, J. Hrabe and S. Hrabetova. Anomalous Extracellular Diffusion in Rat Cerebellum, Biophysical Journal, 108:2384-2395, 2015. 